\title{
EXACT SOLUTIONS TO (3+1) CONFORMABLE TIME FRACTIONAL JIMBO-MIWA, ZAKHAROV-KUZNETSOV AND MODIFIED ZAKHAROV-KUZNETSOV EQUATIONS
}

\author{
ALPER KORKMAZ
}

\begin{abstract}
Exact solutions to conformable time fractional $(3+1)$ - dimensional equations are derived by using the modified form of the Kudryashov method. The compatible wave transformation reduces the equations to an ODE with integer orders. The predicted solution of the finite series of a rational exponential function is substituted into this ODE. The resultant polynomial equation is solved by using algebraic operations. The method works for the Jimbo-Miwa, the Zakharov-Kuznetsov and the modified Zakharov-Kuznetsov equations in conformable time fractional forms. All the solutions are expressed in explicit forms.
\end{abstract}

\section{InTRODUCTION}

The particular integer ordered form of the time fractional Jimbo-Miwa (JM) equation of the type

$$
\eta_{x x x y}+p \eta_{y} \eta_{x x}+q \eta_{x} \eta_{x y}+r D_{\tau}^{\alpha} \eta_{y}-s \eta_{x z}=0
$$

where $D_{\tau}^{\alpha}$ is the $\alpha$.th order fractional derivative operator with respect to $\tau$ is proposed in [1] as the second member of an integrable systems of the KP-hierarchy even though it is not successful to pass the integrability tests[2,3]. Some multi-soliton solutions are determined by bilinear form with an homoclinic test method[3]. These multi-soliton solutions are different from the multi-solitons described by using homogeneous balance and a Bäcklund transformation[4]. Some exact solutions in the forms of solitary waves, periodic waves and variable separation solution to the JM equation are derived by the aid of improved mapping approach[5].The generalized tanh method can be used to generate non traveling-solitonic and moving solitary wave type exact solutions[6]. $\dddot{i} i \frac{1}{2} z \dddot{z} i \underset{i}{2}$ and Aslan also determine some exact solutions to the JM equation by using the method of exp-function[7]. The exact solutions to space-time fractional version of the JM equation are proposed in [8] by using generalized Bernoulli equation method.

The time fractional Zakharov-Kuznetsov (KZ) equation is of the form

$$
D_{\tau}^{\alpha} \eta+p \eta \eta_{x}+q \eta_{z z z}+r \eta_{x x z}+s \eta_{y y z}=0
$$

where $p, q, r$ and $s$ are real parameters. The original form of the equation has been proposed to define three dimensional ion-sound solitons in a magnetized plasma with a low pressure[9]. The three conservation laws describing mass, momentum and center mass are also formulated in the same study. The ZK equation also admits the ellipsoidal and plane type solitons[10]. Some solutions of the cnoidal, periodic, singular periodic, solitary wave and non topological soliton forms are constructed by using the extended hyperbolic tangent, $G^{\prime} / G$ and ansatz methods[11]. The traveling wave solitons in various forms are derived in Ebadi et al.'s study by implementing the exp-function, modified F-expansion and $G^{\prime} / G$ methods[12]. Zhang and Zhou [13] obtains kink, antikink, solitary wave and periodic solutions to the ZK equation in general form by employing the bifurcation theory.

When the nonlinearity of the ZK equation is increased to three, the equation is named as the modified ZK $(\mathrm{mZK})$ equation and the conformable time fractional form of the equation becomes

$$
D_{\tau}^{\alpha} \eta+p \eta^{2} \eta_{x}+q \eta_{z z z}+r \eta_{x x z}+s \eta_{y y z}=0
$$

Liang[14] derived some exact solutions in forms of some trigonometric and hyperbolic functions to the mZK equation by using modified simple equation method. More than twenty exact solutions of the mZK equation are derived by using enhanced $G^{\prime} / G$ method in [15]. The fractional forms of both equations are solved exactly by using improved sub equation adapted for fractional cases[16].

In the present study, finite series of a rational exponential function types solutions are derived for the three dimensional fractional PDEs in conformable sense listed above. All the solutions are expressed explicitly. Before explaining the used procedure, some significant descriptions and calculus properties of the conformable derivative are summarized below.

2010 Mathematics Subject Classification. Primary 35C07;35R11;35Q53. PACS: 02.30.Jr, 02.70.Wz, 47.35.Fg.

Key words and phrases. Fractional (3+1)- dimensional Jimbo-Miwa equation; Fractional modified Zakharov-Kuznetsov equation; Modified Kudryashov method; Fractional Zakharov-Kuznetsov equation; Exact solutions. 


\section{Preliminaries}

Consider a function $\zeta=\zeta(\tau)$ defined in the positive half space and $\alpha$ be number $\in(0,1]$. Then, the conformable derivative of $\zeta$ for $\tau>0$ is defined as

$$
D_{\tau}^{\alpha}(\zeta(\tau))=\lim _{h \rightarrow 0} \frac{\zeta\left(\tau+h \tau^{1-\alpha}\right)-\zeta(\tau)}{h}, \tau>0, \alpha \in(0,1]
$$

for $\zeta:[0, \infty) \rightarrow \mathbb{R}[17]$. Even though this definition of the fractional derivative is pretty new, various important properties such as derivative of multiplication and division are defined clearly. The fundamental properties of the conformable derivative required to solve fractional PDEs are summarized below.

Theorem 1. Let $\zeta=\zeta(\tau)$ and $\eta=\eta(\tau)$ be two $\alpha$-differentiable functions for $\alpha \in(0,1]$. Then

- $D_{\tau}^{\alpha}\left(c_{1} \zeta+c_{2} \eta\right)=c_{1} D_{\tau}^{\alpha}(\zeta)+c_{2} D_{\tau}^{\alpha}(\eta)$

- $D_{\tau}^{\alpha}\left(\tau^{p}\right)=p \tau^{p-\alpha}, \forall p \in \mathbb{R}$

- $D_{\tau}^{\alpha}(\zeta(\tau))=0$, when $\zeta(\tau)=c_{3}$ is constant

- $D_{\tau}^{\alpha}(\zeta \eta)=\zeta D_{\tau}^{\alpha}(\eta)+\eta D_{\tau}^{\alpha}(\zeta)$

- $D_{\tau}^{\alpha}\left(\frac{\zeta}{\eta}\right)=\frac{\zeta D_{\tau}^{\alpha}(\eta)-\eta D_{\tau}^{\alpha}(\zeta)}{\eta^{2}}$

- $D_{\tau}^{\alpha}(\zeta)(\tau)=\tau^{1-\alpha} \frac{d \zeta}{d \tau}$

for all real $c_{1}, c_{2}, c_{3}[18,19]$.

The significant properties covering Laplace transform, derivative of composite functions and Gronwall' inequality are defined in $[20,18]$.

Theorem 2. Let $\zeta$ be a differentiable and $\alpha$-conformable differentiable function and $\eta$ also be defined defined in the range of $\zeta$ and be differentiable. Then,

$$
D_{\tau}^{\alpha}(\zeta \circ \eta)=\left.\tau^{1-\alpha} \eta^{1-\alpha} \eta^{\prime} D_{\tau}^{\alpha} \zeta(t)\right|_{t=\eta(\tau)}
$$

where' denotes the derivative with respect to $\tau[18]$.

\section{The Modified Kudryashov Method}

Let $P$ be

$$
P\left(\eta, \eta_{\tau}^{\alpha}, \eta_{x}, \eta_{y}, \eta_{z}, \eta_{\tau \tau}^{\alpha}, \eta_{x x}, \ldots\right)=0
$$

where $\eta=\eta(x, y, z, \ldots, \tau)$ and $\alpha \in(0,1]$ be the fractional derivative order. The transformation

$$
\eta(x, y, z, \tau)=\eta(\xi), \xi=a x+b y+c z-\frac{\nu}{\alpha} \tau^{\alpha}
$$

converts (3.1) to an ODE for new variable $\xi$

$$
Q\left(\eta, \eta^{\prime}, \eta^{\prime \prime}, \ldots\right)=0
$$

where the prime $\left({ }^{\prime}\right)$ indicates the derivative operator $\frac{d}{d \xi}$ of $\eta$ with respect to $\xi[21]$.

Assume that the equation (3.3) has a solution of the form

$$
\eta(\xi)=a_{0}+a_{1} H(\xi)+a_{2} H^{2}(\xi)+\ldots a_{n} H^{n}(\xi)
$$

for a finite $n$ with all $a_{i}, 0 \leq i \leq n$ and $a_{n} \neq 0$. The procedure start by determining the degree of the polynomial type series $n$ by balancing the non linear term and the highest order derivative term. The function $H$ is required to satisfy the first-order ODE

$$
H^{\prime}(\xi)=H(\xi)(H(\xi)-1) \ln A
$$

Thus, $H(\xi)$ is determined as

$$
H(\xi)=\frac{1}{1+d A^{\xi}}
$$

where $d$ and $A$ are non-zero constants with the conditions $A>0$ and $A \neq 1$.

Substituting the predicted solution (3.4) and its derivatives into (3.3) gives a polynomial of $H(\xi)$. All the coefficients of the powers of $H(\xi)$ and the constant term are equated to zero. The resultant algebraic equation system is solved for $a_{0}, a_{1}, a_{2}, \ldots a_{n}$ and the other constants used in the wave transformation (3.2). This method is explained in details in [22]. 


\section{Solutions to $(3+1)$-Dimensional JM EQuATion}

The JM equation given in the (1.1) is reduced to

$$
a^{3} b \eta^{(i v)}+p a^{2} b \eta^{\prime \prime} \eta^{\prime}+q a^{2} b \eta^{\prime} \eta^{\prime \prime}-\nu b r \eta^{\prime \prime}-s a c \eta^{\prime \prime}=0
$$

by using the compatible form of the transformation (3.2). Rearrangement of the last equation by integrating once gives

$$
a^{3} b \eta^{\prime \prime \prime}+\frac{(p+q)}{2} a^{2} b\left(\eta^{\prime}\right)^{2}-(\nu b r+s a c) \eta^{\prime}=K
$$

where $K$ stands for the constant of integration. The balance between the non linear and the highest ordered terms gives $n=1$. Accordingly, the predicted solution (3.4) should be

$$
\eta(\xi)=a_{0}+a_{1} H(\xi)
$$

where $a_{0}$ and $a_{1}$ are the constants to be determined. Substituting this solution and its derivatives into (1.1) leads

$$
\begin{aligned}
& \left(6 a^{3} b a_{1}(\ln (A))^{3}+1 / 2 a^{2} b a_{1}^{2}(\ln (A))^{2} p+1 / 2 a^{2} b a_{1}^{2}(\ln (A))^{2} q\right) H^{4}(\xi) \\
& +\left(-12 a^{3} b a_{1}(\ln (A))^{3}-a^{2} b a_{1}^{2}(\ln (A))^{2} p-a^{2} b a_{1}^{2}(\ln (A))^{2} q\right) H^{3}(\xi) \\
& +\left(7 a^{3} b a_{1}(\ln (A))^{3}+1 / 2 a^{2} b a_{1}^{2}(\ln (A))^{2} p+1 / 2 a^{2} b a_{1}^{2}(\ln (A))^{2} q-a_{1} \ln (A) s a c-a_{1} \ln (A) b r \nu\right) H^{2}(\xi) \\
& +\left(-a^{3} b a_{1}(\ln (A))^{3}+a_{1} \ln (A) s a c+a_{1} \ln (A) b r \nu\right) H(\xi)-K=0
\end{aligned}
$$

It is clear that a $H(\xi)$ should be nonzero. Hence, the coefficients of all powers of $H(\xi)$ and $K$ should be zero. Thus, the algebraic system of equations

$$
\begin{array}{r}
-a^{3} b a_{1}(\ln (A))^{3}+a_{1} \ln (A) s a c+a_{1} \ln (A) b r \nu=0 \\
7 a^{3} b a_{1}(\ln (A))^{3}+1 / 2 a^{2} b a_{1}^{2}(\ln (A))^{2} p+1 / 2 a^{2} b a_{1}^{2}(\ln (A))^{2} q-a_{1} \ln (A) s a c-a_{1} \ln (A) b r \nu=0 \\
-12 a^{3} b a_{1}(\ln (A))^{3}-a^{2} b a_{1}^{2}(\ln (A))^{2} p-a^{2} b a_{1}{ }^{2}(\ln (A))^{2} q=0 \\
6 a^{3} b a_{1}(\ln (A))^{3}+1 / 2 a^{2} b a_{1}^{2}(\ln (A))^{2} p+1 / 2 a^{2} b a_{1}{ }^{2}(\ln (A))^{2} q=0
\end{array}
$$

Solving (4.5) for $a_{0}, a_{1}, a, b, c, \nu$ gives

$$
\begin{aligned}
a_{1} & =-12 \frac{a \ln (A)}{p+q} \\
c & =\frac{b\left(a^{3}(\ln (A))^{2}-\nu r\right)}{a s}
\end{aligned}
$$

for arbitrary choices of $a_{0}, a, b, \nu$ and $K=0$. The formed solution

$$
\eta(\xi)=a_{0}-12 \frac{a \ln (A)}{(p+q)\left(1+d A^{\xi}\right)}
$$

gives

$$
\eta_{1}(x, y, z, \tau)=a_{0}-12 \frac{a \ln (A)}{(p+q)\left(1+d A^{a x+b y+\frac{b\left(a^{3}(\ln (A))^{2}-\nu r\right)}{a s} z-\nu \frac{\tau^{\alpha}}{\alpha}}\right)}
$$

for arbitrarily chosen $a_{0}, a, b, \nu$. It must be noted that the system (4.5)has three more solutions for $a_{1} \neq 0$ but at least one of $a, b, c, \nu$ are zero in those solution sets. That's why these solutions are not reported here.

\section{Solutions to $(3+1)$-Dimensional ZK EQUATion}

The wave transformation (3.2) reduces the fractional ZK equation (1.2) to

$$
-\nu \eta^{\prime}+p a \eta \eta^{\prime}+q c^{3} \eta^{\prime \prime \prime}+r a^{2} c \eta^{\prime \prime \prime}+s b^{2} c \eta^{\prime \prime \prime}=0
$$

where' denotes $\frac{d}{d \xi}$. Integrating both sides of this equation converts it to

$$
-\nu \eta+\frac{p a}{2} \eta^{2}+\left(q c^{3}+r a^{2} c+s b^{2} c\right) \eta^{\prime \prime}=K
$$

with integration constant $K$. The balance of $\eta^{2}$ and $\eta^{\prime \prime}$ gives the compatible $n$ as 2 . Hence, the predicted solution must be in the form $\eta(\xi)=a_{0}+a_{1} H(\xi)+a_{2} H^{2}(\xi)$. Substituting this solution into (5.2) yields 


$$
\begin{aligned}
& \left(1 / 2 a p a_{2}{ }^{2}+6 b^{2} c s a_{2}(\ln (A))^{2}+6 a^{2} c r a_{2}(\ln (A))^{2}+6 c^{3} q a_{2}(\ln (A))^{2}\right) H^{4}(\xi) \\
& +\left(2 a^{2} c r a_{1}(\ln (A))^{2}-10 a^{2} c r a_{2}(\ln (A))^{2}+2 b^{2} c s a_{1}(\ln (A))^{2}-10 b^{2} c s a_{2}(\ln (A))^{2}+2 c^{3} q a_{1}(\ln (A))^{2}\right. \\
& \left.-10 c^{3} q a_{2}(\ln (A))^{2}+a p a_{1} a_{2}\right) H^{3}(\xi) \\
& +\left(-\nu a_{2}+1 / 2 a p a_{1}^{2}+4 a^{2} c r a_{2}(\ln (A))^{2}+4 b^{2} c s a_{2}(\ln (A))^{2}-3 a^{2} c r a_{1}(\ln (A))^{2}-3 b^{2} c s a_{1}(\ln (A))^{2}\right. \\
& \left.+a p a_{0} a_{2}+4 c^{3} q a_{2}(\ln (A))^{2}-3 c^{3} q a_{1}(\ln (A))^{2}\right) H^{2}(\xi) \\
& +\left(a^{2} c r a_{1}(\ln (A))^{2}+b^{2} c s a_{1}(\ln (A))^{2}+c^{3} q a_{1}(\ln (A))^{2}+a p a_{0} a_{1}-\nu a_{1}\right) H(\xi)-\nu a_{0}+1 / 2 a p a_{0}{ }^{2}-K=0
\end{aligned}
$$

The solution of this system for $a_{0}, a_{1}, a_{2}, a, b, c, \nu$ gives two different solutions as

$$
\begin{aligned}
a_{1} & =-6 \frac{a p a_{0}{ }^{2}+2 K}{a_{0} a p} \\
a_{2} & =6 \frac{a p a_{0}^{2}+2 K}{a_{0} a p} \\
b & = \pm \frac{1}{\ln (A)} \sqrt{-1 / 2 \frac{2(\ln (A))^{2} a^{2} c r a_{0}+2(\ln (A))^{2} c^{3} q a_{0}+a p a_{0}^{2}+2 K}{c s a_{0}}} \\
\nu & =-1 / 2 \frac{-a p a_{0}^{2}+2 K}{a_{0}}
\end{aligned}
$$

for arbitrary constants $a_{0}, a, c$ and $K$. Thus, the solution of (5.2) is determined as

$$
\eta(\xi)=a_{0}-6 \frac{a p a_{0}^{2}+2 K}{a_{0} a p} \frac{1}{1+d A^{\xi}}+6 \frac{a p a_{0}^{2}+2 K}{a_{0} a p} \frac{1}{\left(1+d A^{\xi}\right)^{2}}
$$

arbitrary $a_{0}$ and $K$. The solution of the conformable time fractional ZK equation (1.2) is expressed as

$$
\eta_{2,3}(x, y, z, \tau)=a_{0}-6 \frac{a p a_{0}^{2}+2 K}{a_{0} a p} \frac{1}{1+d A^{a x+b y+c z-\nu \frac{\tau^{\alpha}}{\alpha}}}+6 \frac{a p a_{0}^{2}+2 K}{a_{0} a p} \frac{1}{\left(1+d A^{a x+b y+c z-\nu \frac{\tau^{\alpha}}{\alpha}}\right)^{2}}
$$

where $a_{0}, a, c$ arbitrary $b, \nu$ are as given in (5.4).

\section{Solutions to $(3+1)$-Dimensional mZK EQUATION}

The modified form of the ZK equation in the time fractional form (1.3) is reduced to

$$
-\nu \eta^{\prime}+a \frac{p}{3}\left(\eta^{3}\right)^{\prime}+\left(q c^{3}+r a^{2} c+s b^{2} c\right) \eta^{\prime \prime \prime}=0
$$

where' denotes $\frac{d}{d \xi}$. Integrating this equation once converts it to

$$
-\nu \eta+a \frac{p}{3} \eta^{3}+\left(q c^{3}+r a^{2} c+s b^{2} c\right) \eta^{\prime \prime}=K
$$

with the integration constant $K$. The balance between $\eta^{3}$ and $\eta^{\prime \prime}$ gives $n=1$. Thus, the solution is formed as

$$
\eta(\xi)=a_{0}+a_{1} H(\xi)
$$

for a nonzero $a_{1}$. Substituting this predicted solution and its derivative into (6.2), a polynomial equation of $H(\xi)$ of the form

$$
\begin{aligned}
& \left(1 / 3 a p a_{1}^{3}+2 a^{2} c r a_{1}(\ln (A))^{2}+2 b^{2} c s a_{1}(\ln (A))^{2}+2 c^{3} q a_{1}(\ln (A))^{2}\right) H^{3}(\xi) \\
& +\left(-3 a^{2} c r a_{1}(\ln (A))^{2}-3 b^{2} c s a_{1}(\ln (A))^{2}-3 c^{3} q a_{1}(\ln (A))^{2}+a p a_{0} a_{1}^{2}\right) H^{2}(\xi) \\
& +\left(a^{2} c r a_{1}(\ln (A))^{2}+b^{2} c s a_{1}(\ln (A))^{2}+c^{3} q a_{1}(\ln (A))^{2}+a p a_{0}{ }^{2} a_{1}-\nu a_{1}\right) H(\xi)+1 / 3 a p a_{0}{ }^{3}-\nu a_{0}-K=0
\end{aligned}
$$


is obtained. This algebraic system has two different solutions satisfying the condition $a_{1} \neq 0$ and can be written in the form

$$
\begin{aligned}
a_{0} & = \pm 3 \frac{c \ln (A)\left(a^{2} r+b^{2} s+c^{2} q\right)}{a p} \frac{1}{\sqrt{-\frac{6 r a^{2} c+6 s b^{2} c+6 q c^{3}}{a p}}} \\
a_{1} & = \pm \sqrt{-\frac{6 r a^{2} c+6 s b^{2} c+6 q c^{3}}{a p}} \ln (A) \\
\nu & =-\frac{1}{2}(\ln (A))^{2} c\left(a^{2} r+b^{2} s+c^{2} q\right)
\end{aligned}
$$

for arbitrarily chosen $a, b, c$ and $K=0$. Thus, the solution to (6.2) is constructed as

$$
\eta(\xi)= \pm 3 \frac{c \ln (A)\left(a^{2} r+b^{2} s+c^{2} q\right)}{a p} \frac{1}{\sqrt{-\frac{6 r a^{2} c+6 s b^{2} c+6 q c^{3}}{a p}}} \pm \sqrt{-\frac{6 r a^{2} c+6 s b^{2} c+6 q c^{3}}{a p} \ln (A) \frac{1}{1+d A^{\xi}}}
$$

with the condition $\frac{\left(q c^{2}+r a^{2}+s b^{2}\right) c}{a p}<0, a p \neq 0$. Hence, the solutions of the (1.3) are of the form

$$
\begin{aligned}
\eta_{4,5}(x, y, z, \tau) & = \pm 3 \frac{c \ln (A)\left(a^{2} r+b^{2} s+c^{2} q\right)}{a p} \frac{1}{\sqrt{-\frac{6 r a^{2} c+6 s b^{2} c+6 q c^{3}}{a p}}} \\
& \pm \sqrt{-\frac{6 r a^{2} c+6 s b^{2} c+6 q c^{3}}{a p}} \ln (A) \frac{1}{1+d A^{a x+b y+c z+\frac{1}{2}(\ln (A))^{2} c\left(a^{2} r+b^{2} s+c^{2} q\right) \frac{\tau^{\alpha}}{\alpha}}}
\end{aligned}
$$

\section{Conclusion}

The method of Kudryashov in modified form is implemented to derive the exact solutions to (3+1)-dimensional conformable time fractional JM, ZK and mZK equations. The valid and compatible traveling wave transformation reduces these equations to integer ordered ODEs. The predicted solution of the finite series form of a rational exponential function is substituted into the resultant ODEs. The algebraic operations are used to determine the relations between the coefficients originated from both the equations and the transformation. Once these relations are determined, the traveling wave type solutions in three dimensions are developed explicitly.

\section{REFERENCES}

[1] Jimbo, M., \& Miwa, T. (1983). Solitons and infinite dimensional Lie algebras. Publications of the Research Institute for Mathematical Sciences, 19(3), 943-1001.

[2] Cao, B. (2010). Solutions of Jimbo-Miwa Equation and Konopelchenko-Dubrovsky Equations. Acta applicandae mathematicae, $112(2), 181-203$.

[3] Xu, Z., \& Chen, H. (2015). Cross-kink multi-soliton solutions for the (3+ 1)-D Jimbo-Miwa equation. International Journal of Numerical Methods for Heat \& Fluid Flow, 25(1), 19-24.

[4] Jie-Fang, Z., \& Feng-Min, W. (2002). B'acklund transformation and multiple soliton solutions for the (3+ 1)-dimensional Jimbo-Miwa equation. Chinese Physics, 11(5), 425.

[5] Ma, S. H., Fang, J. P., \& Zheng, C. L. (2009). New exact solutions for the (3+ 1)-dimensional Jimbo-Miwa system. Chaos, Solitons \& Fractals, 40(3), 1352-1355.

[6] Hong, W., \& Oh, K. S. (2000). New solitonic solutions to a (3+1)-dimensional Jimbo-Miwa equation. Computers \& Mathematics with Applications, 39(5), 29-31.

[7] $\dddot{i}_{i} \frac{1}{2}$ ziï $\frac{1}{2}$, T., \& Aslan, $\dddot{i}_{i} \frac{1}{2}$. (2008). Exact and explicit solutions to the (3+1)-dimensional Jimbo-Miwa equation via the Expfunction method. Physics Letters A, 372(47), 7011-7015.

[8] Kolebaje, O. T., \& Popoola, O. O. (2014). Exact Solution of Fractional STO and Jimbo-Miwa Equations with the Generalized Bernoulli Equation Method. The African Review of Physics, 9.

[9] Zakharov, V. E., \& Kuznetsov, E. A. (1974). Three dimensional solitons. Zh. Eksp. Teoret. Fiz., 66, 594-597.

[10] Mace, R. L., \& Hellberg, M. A. (2001). The Korteweg-de Vries-Zakharov-Kuznetsov equation for electron-acoustic waves. Physics of Plasmas (1994-present), 8(6), 2649-2656.

[11] Matebese, B. T., Adem, A. R., Khalique, C. M., \& Biswas, A. (2011). Solutions of Zakharov-Kuznetsov equation with power law nonlinearity in $(1+3)$ dimensions. Physics of Wave Phenomena, 19(2), 148-154.

[12] Ebadi, G., Mojaver, A., Milovic, D., Johnson, S., \& Biswas, A. (2012). Solitons and other solutions to the quantum ZakharovKuznetsov equation. Astrophysics and Space Science, 341(2), 507-513.

[13] Zhang, W., \& Zhou, J. (2012). Traveling Wave Solutions of a Generalized Zakharov-Kuznetsov Equation. ISRN Mathematical Analysis, 2012, 1-10.

[14] Liang, Y. (2014). Exact solutions of the (3+ 1)-dimensional modified KdV-Zakharov-Kuznetsev equation and Fisher equations using the modified simple equation method. Journal of Interdisciplinary Mathematics, 17(5-6), 565-578.

[15] Islam, M. H., Khan, K., Akbar, M. A., \& Salam, M. A. (2014). Exact traveling wave solutions of modified KdV-ZakharovKuznetsov equation and viscous Burgers equation. SpringerPlus, 3(1), 1-9.

[16] Sahoo, S., \& Ray, S. S. (2015). Improved fractional sub-equation method for (3+ 1)-dimensional generalized fractional KdVZakharov-Kuznetsov equations. Computers \& Mathematics with Applications, 70(2), 158-166.

[17] Khalil, R., Al Horani, M., Yousef, A., \& Sababheh, M., A new definition of fractional derivative,bJournal of Computational and Applied Mathematics (2014), 264, 65-70. 
[18] Atangana, A., Baleanu, D., \& Alsaedi, A., New properties of conformable derivative, Open Mathematics (2015) , 13(1), 1-10.

[19] $\dddot{i} i \frac{1}{2}$ enesiz, Y., Baleanu, D., Kurt, A., \& Tasbozan, O., New exact solutions of Burgers' type equations with conformable derivative, Waves in Random and Complex Media (2016), 1-14.

[20] Abdeljawad, T., On conformable fractional calculus,Journal of computational and Applied Mathematics (2015), 279, 57-66.

[21] Eslami, M., \& Rezazadeh, H., The first integral method for Wu-Zhang system with conformable time-fractional derivative, Calcolo (2015), 1-11.

[22] Kudryashov, N. A., One method for finding exact solutions of nonlinear differential equations, Communications in Nonlinear Science and Numerical Simulation (2012), 17(6), 2248-2253.

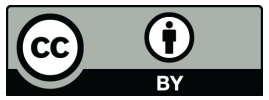

(C)2016 by the author; licensee Preprints, Basel, Switzerland. This article is an open access article distributed under the terms and conditions of the Creative Commons Attribution (CC BY) license (http://creativecommons.org/licenses/by/4.0/).

Alper Korkmaz: Department of Mathematics, Çankiril Karatekin University, 18100 Çankirit, Turkey

E-mail address: korkmazalper@yandex.com 